\title{
HIV/AIDS testing and counselling
}

\author{
A. C. CRAMPIN ${ }^{*}{ }^{* *} \&$ H. DAMISONI $\dagger$ \\ *Karonga Prevention Study, PO Box 46, Chilumba, Karonga District, \\ Malawi \\ **London School of Hygiene and Tropical Medicine, Keppel Street, \\ London WC1E 7HT, UK \\ $\dagger$ National AIDS Secretariat, Ministry of Health and Population, PO \\ Box 30622, Capital City, Lilongwe 3, Malawi
}

\section{The need for HIV testing: clinical, surveillance, research}

In some parts of the world the human immunodeficiency virus and the acquired immune deficiency syndrome (HIV/AIDS) has become the most common in-patient diagnosis amrngst both adults and children. Many countries, although till now relatively spared, are likely to see a huge increase in prevalence of infection and manifestation of disease over the next few years. The daily clinical practice of health care workers (HCWs) will therefore be affected by the necessity to be familiar with the diagnosis of HIV-related conditions and by situations in which they feel it would be beneficial for an individual to know his or her HIV status. The individual may be healthy or may be suffering from what could be an HIV-related disease. HCWs may also be approached by individuals, healthy or sick, asking for HIV testing. Reasons for seeking an HIV test vary and may include concerns over current state of health, concerns over high-risk behaviour (long standing or a particular event), or marriage plans. ${ }^{1}$ The HCW should thus be equipped with the skills to counsel and to arrange an HIV test in a variety of circumstances.

HIV testing is a cornerstone to preventive strategies, as the knowledge of HIV status can have a profound effect on behaviour. Those already infected may change their behaviour in order to reduce transmission to others and those found negative may chamge their behaviour if they feel they have been given a second chance. Using testing to facilitate these changes in behaviour requires effective counselling and provision of support to implement strategies to reduce risk, such as the use of condoms, and the provision of additional information on safer sex and ongoing counselling.

HIV testing may also be performed for surveillance or clinical research purposes. HIV testing in this context is a very sensitive issue. For surveillance it is most commonly done anonymously. For example, in programmes where HIV sero-prevalence surveys are undertaken in the antenatal clinic, the testing is done on 'unlinked' anonymous samples, i.e. after

\footnotetext{
Correspondence to: A. C. Crampin, Karonga Prevention Study, PO Box 46, Chilumba, Karonga District, Malawi.
} 
all identifiers that can link a particular result to an individual have been removed. In many countries this is considered sufficiently protective of the individual to allow testing without consent as the result can never be traced back.

When people (usually patients) are tested for research purposes, it requires careful consideration to select a policy that fits the particular setting. Some research groups undertake 'informed consent without disclosure', i.e. they ask for consent to test the subject's sample, assure them of confidentiality and do not report the result to the subject. This is the most common in community studies on healthy individuals. Others counsel the subjects and report the results to them. In a research setting it is important to ensure that the individual will benefit from knowing their HIV status before selectting the latter policy. There therefore needs to be adequate access to support and post-test counselling and for those who are HIV positive, there needs to be access to a good standard of care.

\section{HIV TESTING-HOW CAN IT BENEFIT THE INDIVIDUAL}

Testing for antibody to HIV is different from many other clinical tests due to its social and ethical implications. ${ }^{2}$ In medical terms there may be little that can be offered by way of treatment in the poorest parts of the world. In addition HIV is a stigmatizing infection, because of its association with sexual activity. It is difficult to predict an individual's prognosis and there are implications for the individual's sexual partners, their unborn children and their social situation. From this, it may seem that HIV testing holds little appeal for an individual, particularly if they suspect they may be found positive. On the other hand, they may realize that knowledge of their HIV status allows them to think and plan realistically for their future; making decisions in their life to maximize their health, making decisions on marriage, re-marriage or childbearing, and taking steps to avoid infecting other people. Importantly, whether testing is or is not desirable for an individual cannot be decided by another person, and the counselling process should enable them to come to an informed choice. People who suspect they may be HIV positive may also be reluctant to undergo testing due to concerns about stigmatization, reactions of relatives, or fears over breach of confidentiality. ${ }^{3,4}$ Individuals may be more concerned about the social/support aspects of AIDS than about the medical aspects, and counsellors should appreciate this. ${ }^{5}$

\section{HIV TESTING - THE SETTING}

The setting in which HIV testing is offered will affect the willingness of individuals to seek testing. Specialized clinics and staff may offer a service that is more professional, but the target group may feel that attendance at such a clinic is itself stimgatizing compared with visiting a general health care facility. Different people are likely to prefer different environments for HIV counselling and testing, ${ }^{6}$ and the availability of a variety of options is likely to maximize the uptake in the community. Different approaches to counselling may be used such as, group or couple or individual counselling, the appropriateness of each depending on the individual and their medical and social circumstances.

HIV TESTING-WHAT TEST TO USE

Many tests are available, and their description is not within the scope of this article. The most widely used tests do not identify the presence of the virus itself, but the antibody response to 
the virus. Thus the antibody tests may be negative in early infection (prior to 'seroconversion') before the antibody has risen to a detectable level, or negative late in disease when the immune system has broken down to the extent when antibody production is no longer possible. The important thing is to know the limitations of the testing method employed, its reliability, and sensitivity and specificity. It may be appropriate to repeat positive tests if the individual has not tested positive previously. The test should also be appropriate for the region, as HIV-2 is prevalent in some communities and not in others. Many tests only identify HIV-1 infection. Some rapid tests are available that can be performed at the bedside or in the clinic for quick results but most are still done by laboratory assays requiring a qualified laboratory technician.

\section{Counselling}

As a general principle, all testing should be promoted with a view towards aftercare, with particular consideration to the availability of support services, drugs and condoms. The counsellor should have the facility to refer the individual where appropriate for treatment of sexually transmitted infections or tuberculosis, for psychological support and to self-help groups for people living with HIV or AIDS.

\section{COUNSELLING-THE COUNSELLOR}

People from a variety of backgrounds may become good counsellors. Many counsellors will be HCWs, drawn into it through their normal duties. Sometimes, however, other people may be more suitable particularly for special groups, such as youth or commercial sex workers. Factors such as age and gender and approachability should be taken into consideration, and peers or influential/respected members of the appropriate community may be appropriate.

HCWs may feel that they do not have enough time to counsel well or they may feel inadequately prepared or trained. There are, however, guidelines that can be followed, usually available from National AIDS control programmes or other organizations involved in HIV/ AIDS within a country. It is important that all HCWs are aware of and have access to such materials.

A close knowledge and understanding of the community is required for effective counselling, but it may be difficult for someone to counsel within their own community. A counsellor may feel embarassed discussing sexual practices with friends and neighbours. In addition, the individual being counselled may feel that confidentiality is more likely to be breached if the counsellor is a member of their close community. Counselling by someone of the same sex is generally more comfortable for the individual.

It is important that those who direct Health Services recognize that the counsellors themselves need support in this difficult process. They need training and ongoing support to assist them in dealing with difficult situations. For example, situations may arise in which the counsellor feels that disclosure to other people is appropriate, either to spouses, relatives or medical staff. As a matter of principle, however, the individual being tested should have the right to choose with whom to share their results, and this should be respected. There may be a conflict in some countries where notification to authorities is a legal requirement in which case the counsellor will need to take this into account when helping the individual to decide 
whether they wish to be tested. There are particular challenges when the event precipitating the need for testing involves rape or incest.

The counsellor should ensure that there is a rationale for testing in each case. It should not be done indiscriminately. A good rationale, whether it is the individual's wishes or clinical condition, will make the counselling easier.

\section{PRE- AND POST-TEST COUNSELLING}

Adequate 'pre-test counselling' is very important. The issues that should be covered in a pretest counselling session are summarized in Box 1. Good pre-test counselling will assist the counsellor greatly when it comes to giving a result, as the individual will have been prepared for eventualities. Immediately after the result is given, there may be either great relief or distress. A prepared individual is likely to be able to absorb better what the counsellor is saying and to consider the advice later on.

Box 1. Pre-testing counselling checklist $^{7}$

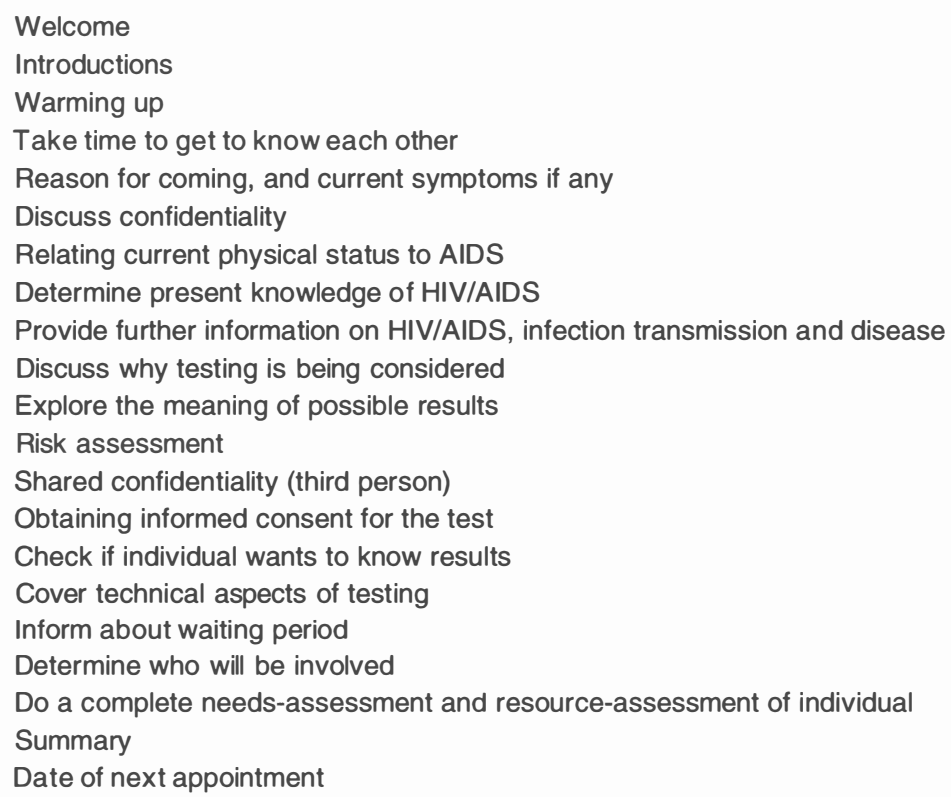

Counselling is an opportunity for education, but it should not be a lecture. Counselling should be culturally appropriate, non-judgmental, take place in privacy and it should be relevant to the individual. It should be appropriate to their educational level. The counsellor should be familiar with local terms for disease and local conceptions of disease causation, in order to be able to explain well how HIV is transmitted and treated. In societies where wasting illnesses are thought to be due to witchcraft or spiritual transgression, being able to explain about HIV in that context is necessary, being sensitive to the individual's beliefs. The counsellor should have adequate knowledge of the transmission, infection and disease. For example, he/she should know the modes of transmission and how to avoid infection, the 
window period during which time an antibody test may be negative, the latent period, the symptoms of the disease and the prognosis and should know what is available locally in terms of support and treatment. Counsellors should not attempt to impose their own attitudes and religious beliefs.

An assessment should be made of the individual's ability to deal with a positive result, and the risk of depression, self-harm or suicide. Whether the person wishes a relative or close friend to share the results with them should be explored, and if he or she seems unlikely to be able to cope with a positive result, or to have inadequate social support, then this should be taken into account when discussing whether the person should undergo testing. The counsellor should be aware of the possible social effects that a positive test results may have in the community and take measures to prevent inadvertent disclosure or breaches of confidentiality. It may be appropriate to counsel and test a married or cohabiting couple together, but the counsellor should understand that there might be subsequent social problems, particularly if there is discordance between the results of the two individuals. In some societies, an HIV positive woman may be divorced or be subject to violence.

A checklist for post-test counselling is presented in Box. 2.

Box 2. Post-test counselling ${ }^{7}$

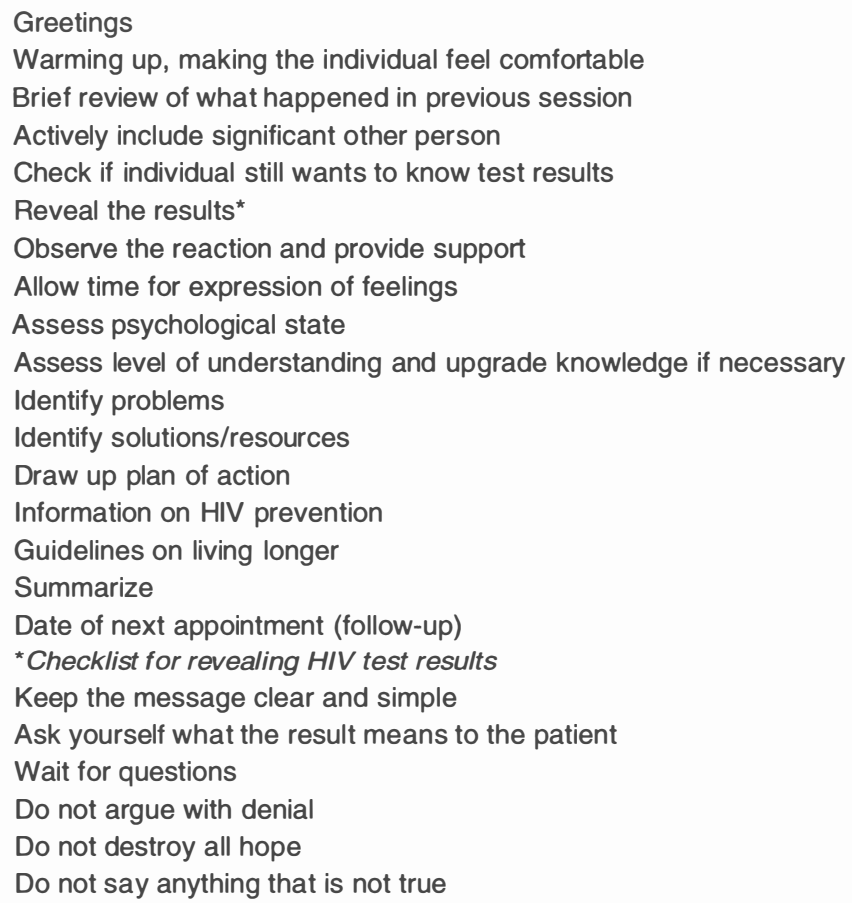

\section{COUNSELLING CHILDREN}

Counselling in the case of HIV testing a child is a difficult issue. In young children, the parent or guardian will need to be counselled, and the counsellor should be aware of the implications 
that a positive result in a young child will have for the mother's HIV status, as mother-tochild-transmission is the most likely route of infection. In older children who may have been sexually active (with or without consent) an assessment should be made as to whether it is culturally and individually appropriate to counsel the child with or without the parent or guardian.

\section{COUNSELLING WITHOUT TESTING}

It may not always be possible or necessary to offer HIV testing when counselling is required. This situation might arise when there is a reasonable certainty of the diagnosis, for example when an individual has clinical features of AIDS. Even without access to testing, counselling may cover many of the same issues, review individual knowledge, conduct a risk assessment and review the level of understanding. The possibility of an HIV related diagnosis should be discussed, given the individual's level of risk and symptoms. Personal risk reduction and the implications of infection may still be covered. The individual may require referral to clinical services for further care and support. It is also appropriate to offer counselling when HIV testing is available, but for some particular reason the individual does not wish to undergo testing, perhaps because of fear or concerns over confidentiality.

ETHICAL ISSUES IN HIV TESTING AND COUNSELLING

\section{Individuals should not be tested for HIV without their informed consent}

'Informed' means not only that they understand that a test for HIV is being conducted, but also that they are educated about HIV and its modes of transmission, and understand the benefits or risks to them of knowing the result and what they can expect from local medical, social and community services should they have a positive result. Confidentiality is a key to the confidence of those being tested. They must feel they can trust the counsellor involved and also that their sample will be handled in a confidential manner. This may require coding of the sample details so that staff handling the transport and processing of the sample and result cannot easily identify the individual. Care must be taken to ensure that there is no opportunity for results to be assigned to the wrong person. People may also be concerned that their test result may be communicated to employers, police, relatives and so on and they must be confident that this will not occur.

HIV-TESTING IN LEPROSY

HIV/AIDS causes a wide variety of dermatological and neurological symptoms and signs, and therefore HIV testing may be appropriate as part of the diagnostic work-up in some patients suspected of having leprosy in areas of high HIV prevalence. Whether HIV infection is a risk factor for leprosy has been investigated in several populations, and with a few exceptions ${ }^{8}$ the answer has been no. Some studies have indicated that HIV may predispose to reactions or relapse. ${ }^{9-12}$ The cumulative evidence indicates that HIV is not an important determinant of leprosy, perhaps because HIV-infected individuals in leprosy endemic areas succumb to the classical AIDS-related conditions before the leprosy bacillus is able to take advantage of the immunosuppressed state to infect the individual or cause disease. HIV testing in leprosy patients is reliable. Reports that there may be false positive HIV results in 
leprosy patients due to cross-reactive antigens have not been borne out by detailed investigations. $^{13}$

All of the issues involved in counselling and testing for HIV apply to patients with leprosy, with the added concern that the occurrence of leprosy and HIV in a single individual may increase the problem of social stigmatization.

\section{References}

${ }^{1}$ Phanuphak P, Muller O, Sarangbin S, Sittatrai W. Knowledge, attitudes and behaviour among HIV-positive and HIV-negative individuals of a confidential HIV counselling and testing centre in Thailand. AIDS, 1994; 8: 13151319.

2 Moss-S, Williams, OE, Hind CR. Counselling for an HIV test. Postgrad Med. J, 1996; 72: 84-66.

3 Coulibaly-D, Msellatic P, Dedy S et al. Attitudes and behaviour of pregnant women towards HIV screening in Abidjan in 1995 and 1996. Sante, 1998; 8: 234-238.

${ }^{4}$ Buwalda P, Kruuijthoff DJ, de-Bruyn M, Hogewoning A. Evaluation of a home-care/counselling AIDS programme in Kgatleng district, Botswana. AIDS-Care, 1994; 6: 153-160.

5 Lie GT, Biswalo PM. Perceptions of appropriate HIV/AIDS counsellors in Arusha and Kilimanjaro regions of Tanzania: implications for hospital counselling. AIDS-Care, 1994: 6: 139-151.

${ }^{6}$ Hong BA, Berger SG. Characteristics of individuals using different HIV/AIDS counseling and testing programs. AIDS, 1994; 8: 259-262.

7 Adapted from CARE Counselling course materials, June 2001, Lilongwe, Malawi.

8 Borgdorff MW, van den Broek J, Chum HJ et al. HIV-1 as a risk factor for leprosy: a case-control study in Tanzania. Int J Lepr, 1993; 61: 556-562.

9 Andrade VL, Moreira-Alves T, Regazzi-Avelleira JC, Bayona M. Prevalence of HIV-1 in leprosy patients in Rio de Janeiro, Brazil. Acta Leprol, 1997; 10: 159-163.

${ }^{10}$ Leinhardt C, Kamate B, Jamet P et al. Effect of HIV infection on leprosy: a three year survey in Bamako, Mali. Int $J$ Lepr, 1996; 64: 383-391.

11 Sekar B, Jayasheela M, Chattopadhya D et al. Prevalence of HIV infection and high risk characteristics among leprosy patients in South India: a case-control study. Int J Lepr, 1994; 62: 527-531.

12 Ponnighaus JM, Mwanjasi LJ, Fine PEM et al. Is HIV a risk factor for leprosy? Int J Lepr, 1991; 59: 221-228.

13 Sterne JAC, Turner AC, Fine PEM et al. Testing for antibody to human immunodeficiency virus type 1 in a population in which mycobacterial diseases are endemic. $J$ Infect Dis, 1995; 172: 543-546. 